\title{
Association between visceral and subcutaneous adiposity and clinicopathological outcomes in non-metastatic clear cell renal cell carcinoma
}

\author{
Roy Mano, MD; ${ }^{*}$ A. Ari Hakimi, MD; ${ }^{*}$ Emily C. Zabor, MS; ${ }^{\dagger}$ Marta A. Bury, MD,s Olivio F. Donati, MD; \\ Christoph A. Karlo, MD,; Wassim M. Bazzi, MD,* Helena Furberg, PhD; ${ }^{\dagger}$ Paul Russo, MD*
}

*Urology Service, Department of Surgery, Memorial Sloan Kettering Cancer Center, New York, NY; 'Department of Epidemiology and Biostatistics, Memorial Sloan Kettering Cancer Center, New York, NY; §Department of Radiology, Memorial Sloan Kettering Cancer Center, New York, NY

Cite as: Can Urol Assoc J 2014;8(9-10):e675-80. http://dx.doi.org/10.5489/cuaj.1979 Published online October 22, 2014.

\section{Abstract}

Introduction: Visceral adiposity has been inconsistently associated with clinicopathologic features and outcomes of clear cell renal cell carcinoma (ccRCC); however, most studies were conducted in non-Western populations. We evaluated the associations between visceral and subcutaneous adiposity and clinicopathological characteristics of non-metastatic ccRCC patients in a Western population.

Methods: The medical records of 220 surgically treated ccRCC patients with documented preoperative body mass index (BMI) and computed tomography (CT) scans were retrospectively reviewed. Nineteen patients with stage IV disease were excluded. Visceral (VFA) and subcutaneous fat area (SFA) were computed from preoperative CT scans. Correlations between obesity measures were assessed with Pearson correlation. Associations between obesity measures and pathologic features were evaluated using logistic regression models adjusted for sex. Overall survival (OS) probabilities were estimated using Cox regression analysis. The log-rank test was used for group comparisons.

Results: The study cohort comprised 150 men and 51 women. Women had higher SFA $(p=0.01)$ but lower VFA $(p<0.001)$ than men. BMI was highly correlated with SFA $(r=0.804)$ and moderately correlated with VFA $(r=0.542)$. SFA and VFA were weakly correlated $(r=0.367)$. An increased BMI was associated with a better OS $(p=0.028)$. When adjusting for sex, neither SFA nor VFA was significantly associated with tumour grade, stage, or OS. Conclusions: Consistent with prior reports, our study suggests that increased $\mathrm{BMI}$ is associated with a better OS for patient with nonmetastatic ccRCC. Despite the high correlation between SFA and BMI, neither SFA nor VFA were significantly associated with tumour stage, grade, or OS in the current study; however, further studies in larger cohorts are required to validate this finding.

\section{Introduction}

Obesity, as measured by body mass index (BMI), is an established risk factor for renal cell carcinoma (RCC). ${ }^{1,2}$ Paradoxically, higher BMI appears to be associated with better prognosis among RCC patients. ${ }^{3,4}$ As BMI is an indirect measure of adiposity that does not distinguish between adipose tissue and lean mass, ${ }^{5}$ other metrics of body size should be considered in relation to RCC prognosis.

Visceral adipose tissue has multiple endocrine, metabolic, and immunological functions and is associated with metabolic syndrome and tumorigenesis. Possible mechanisms for the promotion of tumour development include alterations in adipokine production, insulin and insulin-like growth factor pathways, cancer cell signaling and inflammatory pathways. ${ }^{6}$ Visceral fat has been identified as an independent risk factor and a predictor of outcome in several cancers, including breast, esophageal, colorectal and pancreatic cancers. ${ }^{7-11}$

Altered metabolism has a central role in RCC pathogenesis, possibly explaining the link between obesity and RCC. ${ }^{4,12}$ While the reported effects of BMI on RCC have been uniform, reports concerning the effect of visceral adiposity on the pathological and clinical outcomes of RCC are inconsistent. ${ }^{13-17}$ Moreover, most previous studies evaluating the predictive value of visceral fat in non-metastatic RCC were conducted among Asian populations, which have relatively lower BMIs than Western populations, and may display a different relationship between BMI and body fat. ${ }^{18,19}$

The aim of the current study was to evaluate whether visceral fat area (VFA) and subcutaneous fat area (SFA) were related to tumour characteristics and treatment outcomes in a group of surgically treated, non-metastatic, clear cell RCC (cCRCC) patients from the United States. 
Mano et al.

\begin{tabular}{|c|c|c|c|c|}
\hline & Total $(\mathbf{n}=\mathbf{2 0 1})$ & Male ( $n=150 ; 75 \%)$ & Female ( $n=51 ; 25 \%)$ & $p$ value* \\
\hline Median age (range) & $59.0(34.0-86.0)$ & $60.0(34.0-86.0)$ & $58.0(37.0-79.0)$ & 0.662 \\
\hline Race, n (\%) & & & & 0.010 \\
\hline White & $182(90.5)$ & $141(94.0)$ & $41(80.4)$ & \\
\hline Other & $19(9.5)$ & $9(6.0)$ & $10(19.6)$ & \\
\hline Hypertension, n (\%) & & & & 0.512 \\
\hline Yes & $117(58.2)$ & $85(56.7)$ & $32(62.7)$ & \\
\hline No & $84(41.8)$ & 65 (43.3) & $19(37.3)$ & \\
\hline Hypercholesterolemia, n (\%) & & & & 1.000 \\
\hline Yes & $69(34.3)$ & $52(34.7)$ & $17(33.3)$ & \\
\hline No & $132(65.7)$ & $98(65.3)$ & $34(66.7)$ & \\
\hline Diabetes, n (\%) & & & & 1.000 \\
\hline Yes & $26(12.9)$ & $20(13.3)$ & $6(11.8)$ & \\
\hline No & $175(87.1)$ & $130(86.7)$ & $45(88.2)$ & \\
\hline
\end{tabular}

${ }^{*} p$ value from Fisher's exact test when categorical, and Wilcoxon test when continuous; ccRCC: clear cell renal cell carcinoma.

\section{Methods}

After obtaining Institutional Review Board approval, we retrospectively reviewed the medical records of 220 ccRCC patients, who were surgically treated between 2001 and 2011 at a single institute, and had both a pre-surgical BMI value and CT scan available for review. Nineteen patients with American Joint Committee on Cancer (AJCC) stage IV disease ${ }^{20}$ were excluded from the study, as their weight at diagnosis could have been affected by their disease.

Patient and disease characteristics were recorded. All tumours were identified as cCRCCs and graded according to the Fuhrman grading system..$^{21}$ For data analysis, tumour grade was dichotomized as grade II (low grade) or grades III and IV (high grade). Tumours were measured for their maximal diameter and staged according to the 2002 AJCC TNM classification. ${ }^{20}$ Due to the low number of pathological T2 disease $(n=17)$, we combined the pT1 and pT2 stages and compared them to the pT3 stage in the data analyses.

Presurgical BMI was calculated as patient's weight in kilograms divided by their height in meters squared, and categorized into normal weight (BMl $<25 \mathrm{~kg} / \mathrm{m}^{2}$ ), overweight $\left(25 \mathrm{~kg} / \mathrm{m}^{2} \leq \mathrm{BMI}<30 \mathrm{~kg} / \mathrm{m}^{2}\right)$, and obese $\left(\mathrm{BMI} \geq 30 \mathrm{~kg} / \mathrm{m}^{2}\right)$ according to the World Health Organization classification. ${ }^{22}$ VFA, SFA and total fat area (TFA) measurements were derived from presurgical CT scans at the level of the umbilicus with the patient in the supine position using the ImageJ software, as previously described (Fig. 1). ${ }^{23,24}$ In separate measurement sessions, the subcutaneous and intra-abdominal adipose areas were manually outlined and measured on the basis of predefined Hounsfield unit thresholds (-190 to -30). Measurements of the adipose area were performed by 2 radiologists blinded to the clinical and pathological data. The percentage of visceral fat (VFA\%) was calculated using the formula VFA $\%=($ VFA/TFA $) \times 100$.

Patient characteristics, disease variables and obesity measures were reported using descriptive statistics, and compared between the genders using Fisher's exact test for categorical measures and Wilcoxon rank sum test for continuous variables. Median, minimal and maximal values were calculated for continuous variables; categorical values were described as number and percentage. Inter-reader agreement analysis was performed using the intra-class correlation coefficient. Pearson correlation coefficients were calculated
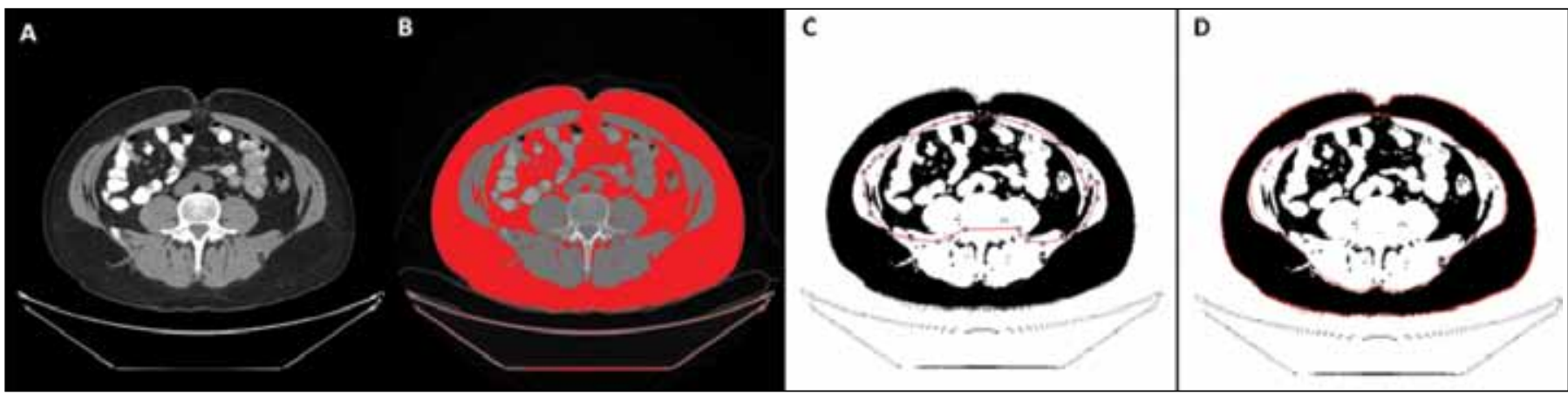

Fig. 1. Measurement of visceral fat area (VFA) and subcutaneous fat area (SFA) using the ImageJ software. (A) A computed tomography scan at the level of the umbilicus; (B) A total fat area is highlighted on the ImageJ software using predefined Hounsfield units (-30 to -190); (C) VFA is manually outlined and measured. (D) Similarly, SFA is manually outlined and measured. 
Table 2. Disease characteristics of 201 ccRCC patients, stratified by sex

\begin{tabular}{|c|c|c|c|c|}
\hline & Total $(n=201)$ & Male ( $=150 ; 75 \%)$ & Female ( $=51 ; 25 \%)$ & $p$ value* \\
\hline Presentation, n (\%) & & & & 0.552 \\
\hline Incidental & $161(80.1)$ & $121(80.7)$ & $40(78.4)$ & \\
\hline Local & $36(17.9)$ & 25 (16.7) & $11(21.6)$ & \\
\hline Systemic & $4(2.0)$ & $4(2.7)$ & $0(0.0)$ & \\
\hline Laterality, n (\%) & & & & 0.198 \\
\hline Left & $102(50.7)$ & $72(48.0)$ & $30(58.8)$ & \\
\hline Right & 99 (49.3) & $78(52.0)$ & $21(41.2)$ & \\
\hline Grade, n (\%) & & & & $<0.001$ \\
\hline 2 & $85(42.3)$ & 50 (33.3) & $35(68.6)$ & \\
\hline 3 & $102(50.7)$ & $87(58.0)$ & $15(29.4)$ & \\
\hline 4 & $14(7.0)$ & $13(8.7)$ & $1(2.0)$ & \\
\hline Median tumour size (range) & $4.2(1.0-15.2)$ & $4.5(1.0-13.1)$ & $3.9(1.5-15.2)$ & 0.169 \\
\hline T stage, n (\%) & & & & 0.160 \\
\hline T1 & $103(51.2)$ & $73(48.7)$ & $30(58.8)$ & \\
\hline T2 & $17(8.5)$ & $11(7.3)$ & $6(11.8)$ & \\
\hline T3 & $81(40.3)$ & $66(44.0)$ & $15(29.4)$ & \\
\hline $\mathrm{N}$ stage, $\mathrm{n}(\%)$ & & & & 1.000 \\
\hline$N X$ & $121(60.2)$ & $90(60.0)$ & $31(60.8)$ & \\
\hline No & $78(38.8)$ & $58(38.7)$ & $20(39.2)$ & \\
\hline N1 & $2(1.0)$ & $2(1.3)$ & $0(0.0)$ & \\
\hline AJCC stage, $\mathrm{n}(\%)$ & & & & 0.130 \\
\hline I & $102(50.7)$ & $72(48.0)$ & $30(58.8)$ & \\
\hline II & $17(8.5)$ & $11(7.3)$ & $6(11.8)$ & \\
\hline III & $82(40.8)$ & $67(44.7)$ & $15(29.4)$ & \\
\hline
\end{tabular}

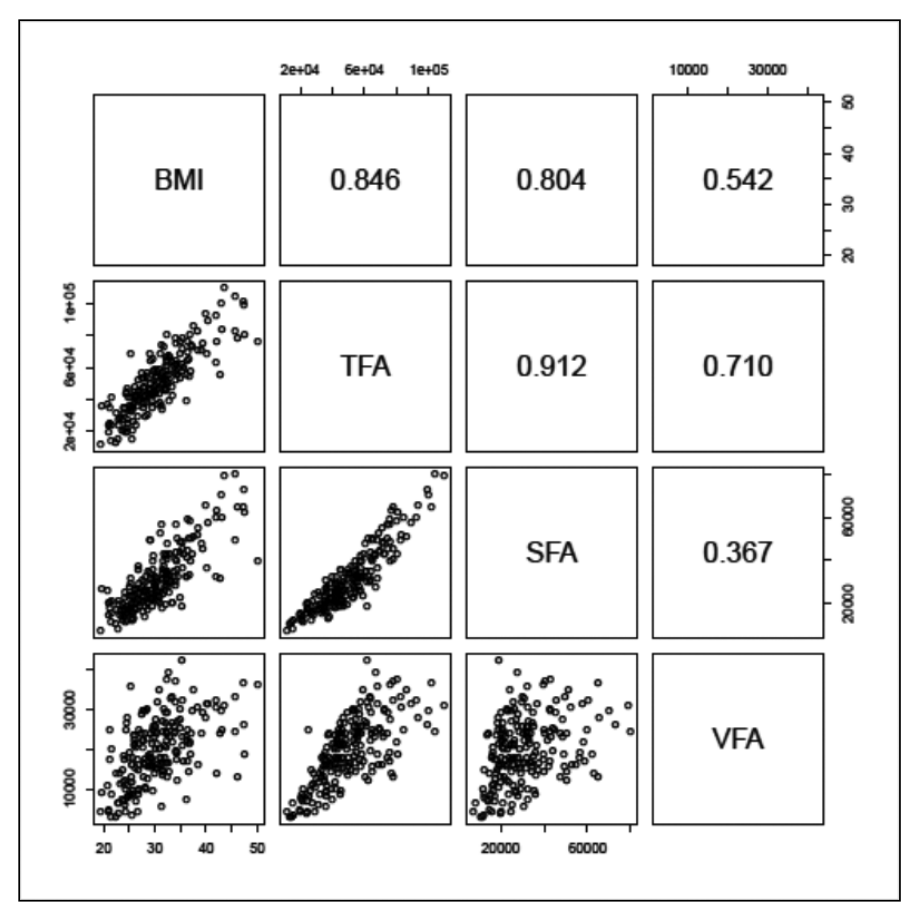

Fig. 2. Scatterplot matrix of the different obesity measures (lower panels) and their associated Pearson correlation values (upper panels). among the different obesity measures and a scatterplot matrix was plotted. To examine the associations between obesity measures and the presence of medical comorbidities, advanced tumour stage (T3) and high-grade disease (III/IV), we used fitted logistic regression models adjusting for sex.

Overall survival (OS) time was calculated from date of surgery to date of death or last contact. Patients still alive or lost to follow-up were censored at the last follow-up date. Associations with OS were assessed using Cox regression, adjusted for sex, and the log-rank test. All statistical analyses were two-sided. Statistical significance was defined as $p<0.05$. Statistical analyses were conducted using SAS v.9.2 (SAS Institute, Cary, NC) and R version 2.13.1 (R Core Development Team, Vienna, Austria), including the "survival" package.

\section{Results}

The analytic cohort included 150 male and 51 female patients with a median age of 59 (interquartile range [IQR]: 51-68) years. Women were more likely to be non-white $(20 \%$ vs. $6 \% ; p=0.010)$ as compared to men (Table 1$)$.

Disease characteristics stratified by sex are summarized in Table 2. Tumour grade was II in 85 patients (42\%) and III/IV in 116 patients (58\%). The median tumour size was $4.2 \mathrm{~cm}$ 
Mano et al.

\begin{tabular}{|c|c|c|c|c|}
\hline & Total $(n=201)$ & Male ( $n=150 ; 75 \%$ ) & Female (n = 51; $25 \%$ ) & $p$ value* \\
\hline BMI category, n (\%) & & & & 0.083 \\
\hline$<25$ & $34(16.9)$ & $20(13.3)$ & $14(27.5)$ & \\
\hline $25-<30$ & $68(33.8)$ & $53(35.3)$ & $15(29.4)$ & \\
\hline$\geq 30$ & $99(49.3)$ & 77 (51.3) & $22(43.1)$ & \\
\hline Median TFA (range) & $48423(11697-109671)$ & $48202(11697-104010)$ & 49888 (13433-109671) & 0.807 \\
\hline Median SFA (range) & 27913 (7377-79789) & 26418 (7377-79789) & 32765 (10685-78819) & 0.010 \\
\hline Median VFA (range) & 19009 (2748-42375) & 21862 (3075-42375) & 15649 (2748-35898) & $<0.001$ \\
\hline Median VFA\% (range) & $39.2(14.9-98.6)$ & $41.9(21.0-68.9)$ & $27.7(14.9-98.6)$ & $<0.001$ \\
\hline
\end{tabular}

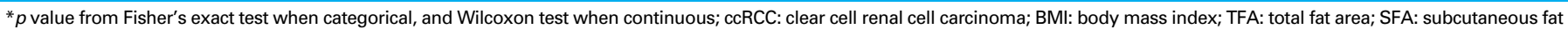
area; VFA: visceral fat area.

(IQR: 3.2-7). Pathological T-stage was T1/T2 in 120 patients $(60 \%)$ and T3 in 81 patients $(40 \%)$. Women were more likely to have grade II disease $(69 \%$ vs. $33 \% ; p<0.001)$ as compared to men.

The inter-reader agreement analysis for the 2 radiologists showed a high level of agreement for all obesity measures performed on the CT scans. The intra-class correlation coefficient was 0.994 (95\% confidence interval [Cl] 0.992-0.995) for TFA, 0.995 (95\% Cl 0.994-0.997) for SFA, and 0.937 $(95 \% \mathrm{Cl} 0.918-0.951)$ for VFA. As a result of the high levels of agreement, the 2 measurements were averaged for all remaining analyses. The median BMI, VFA and SFA were $29.9 \mathrm{~kg} / \mathrm{m}^{2}$ (IQR: 26-33.8), $19009 \mathrm{~mm}^{2}$ (IQR: 14248 24940 ) and $27913 \mathrm{~mm}^{2}$ (IQR: 20 518-37 459), respectively (Table 3). Women had higher SFA $(p=0.010)$ but lower VFA $(p<0.001)$ as compared to men, but did not differ with regard to $\mathrm{BMI}$ or TFA.

BMI was highly correlated with SFA $(r=0.804)$, and moderately correlated with VFA $(r=0.542)$. SFA and VFA were weakly correlated $(r=0.367)$ (Fig. 2$)$. The lowest correlation was seen between BMI and VFA $\%(r=-0.156)$. Correlations were also calculated separately by sex, with similar patterns seen for both men and women.

The association between the obesity measures and medical comorbidities are summarized in Table 4. Hypertension was significantly associated with higher BMI, TFA, VFA, and SFA. Diabetes was significantly associated with higher TFA and VFA.

Table 5 summarizes the obesity measures by tumor stage and grade. The odds of having advanced stage or high-grade disease did not differ significantly with regard to TFA, SFA, VFA, or VFA\% after adjusting for sex (all $p>0.05$ ). Median follow-up time among survivors was 4 years (range: 0.04 9). During follow-up, 26 patients died from various causes. Consistent with prior reports, an increased BMI was associated with a better OS on univariable analysis $(p=0.028)$. However, we found no statistically significant associations between TFA, SFA, VFA, or VFA\% and OS after adjusting for sex (Table 5). Nevertheless, while not statistically significant, there was a trend toward an association between higher SFA and longer OS (HR 0.72, 95\% Cl 0.50-1.05; $p=0.091$ ), suggesting that SFA may show a similar paradoxical relationship with survival as BMI.

\section{Discussion}

The current study examined the associations between different measures of body size in relation to clinicopathological variables among a group of non-metastatic ccRCC patients in the United States. While SFA and BMI were highly correlated, SFA and VFA were poorly correlated and differed by sex, suggesting these obesity measures represent different aspects of body size. No statistically significant associations were observed between SFA or VFA and grade, stage or OS. However, there was a trend toward an association between SFA and OS, similar to the pattern seen with BMI in this and other studies.

Body fat tissue is traditionally distributed in two main compartments, subcutaneous and visceral adipose tissue, each of which has unique cellular, physiological, clini-

Table 4. Associations between obesity measures and medical comorbidities

\begin{tabular}{|c|c|c|c|c|c|c|}
\hline & \multicolumn{2}{|c|}{ Hypertension } & \multicolumn{2}{|c|}{ Hypercholesterolemia } & \multicolumn{2}{|c|}{ Diabetes } \\
\hline & OR $(95 \% \mathrm{CI}) *$ & $p$ Value** & OR $(95 \% \mathrm{Cl}) *$ & $p$ Value** & OR $(95 \% \mathrm{Cl}) *$ & $p$ value** \\
\hline BMI (per 10) & $2.13(1.26-3.59)$ & 0.005 & $1.18(0.73-1.91)$ & 0.501 & $1.46(0.76-2.81)$ & 0.254 \\
\hline TFA (per 10,000) & $1.38(1.16-1.63)$ & $<0.001$ & $0.98(0.84-1.15)$ & 0.831 & 1.29 (1.05-1.59) & 0.017 \\
\hline SFA (per 10,000) & 1.42 (1.13-1.79) & 0.003 & $1.00(0.81-1.23)$ & 0.979 & $1.29(0.99-1.69)$ & 0.060 \\
\hline VFA (per 10,000) & $2.12(1.42-3.16)$ & $<0.001$ & $0.89(0.62-1.29)$ & 0.554 & $1.91(1.12-3.27)$ & 0.018 \\
\hline VFA\% (per 10\%) & $1.16(0.88-1.51)$ & 0.290 & $0.91(0.69-1.19)$ & 0.493 & $1.12(0.78-1.60)$ & 0.533 \\
\hline
\end{tabular}

${ }^{*}$ Adjusted for sex; ${ }^{*} p$ value from logistic regression; BMI: body mass index; TFA: total fat area; SFA: subcutaneous fat area; VFA: visceral fat area; OR: odds ratio; Cl: confidence interval. 


\begin{tabular}{|c|c|c|c|c|c|c|}
\hline & \multicolumn{2}{|c|}{ Advanced stage (T3) } & \multicolumn{2}{|c|}{ High grade (3/4) } & \multicolumn{2}{|c|}{ Overall survival } \\
\hline & OR $(95 \% \mathrm{Cl}) *$ & $p$ Value** & OR $(95 \% \mathrm{Cl}) *$ & $p$ Value** & HR $(95 \% \mathrm{Cl}) *$ & $p$ valuet \\
\hline TFA (per 10 000) & $0.97(0.84-1.13)$ & 0.734 & $0.91(0.78-1.06)$ & 0.218 & $0.83(0.65-1.05)$ & 0.116 \\
\hline SFA (per 10000 ) & $0.98(0.80-1.20)$ & 0.825 & $0.90(0.73-1.11)$ & 0.318 & $0.72(0.50-1.05)$ & 0.091 \\
\hline VFA (per 10000$)$ & $0.97(0.68-1.39)$ & 0.868 & $0.84(0.57-1.22)$ & 0.349 & $0.82(0.49-1.36)$ & 0.446 \\
\hline VFA\% (per 10\%) & $1.05(0.81-1.37)$ & 0.687 & $1.05(0.80-1.37)$ & 0.732 & $1.13(0.80-1.59)$ & 0.503 \\
\hline
\end{tabular}

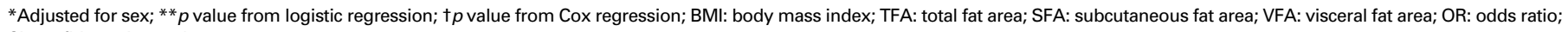
$\mathrm{Cl}$ : confidence interval.

cal and prognostic characteristics. ${ }^{25,26}$ Visceral fat, but not subcutaneous fat, was associated with an increased risk for cardiovascular disease, cancer and all-cause mortality in men. ${ }^{6,25,27}$ Due to its excellent resolution for adipose tissue, CT enables quantitative assessment of visceral and subcutaneous fat. ${ }^{26}$ VFA, as measured at the level of the umbilicus, is highly correlated with the total visceral fat volume, and can be used to evaluate visceral fat accumulation. ${ }^{23}$

Previous reports reveal stronger correlations for BMI with TFA and SFA than with VFA when controlling for age and race. Furthermore, regardless of $\mathrm{BMI}$, women appear to have higher levels of SFA and fat mass than men. $8,10,16,17,28$ Similarly, in the current study the correlation between BMI and VFA $(r=0.542)$ was relatively weak when compared to that of BMI and SFA $(r=0.804)$ and BMI and TFA $(r=0.846)$. In addition, women had higher SFA but lower VFA than men, but did not differ from men with regard to BMI or TFA. The amount of visceral fat differed significantly according to ethnic origin, which brings into question the ability to generalize the findings of previous reports performed on specific study populations. ${ }^{18,19}$ In the current study, $90 \%$ of participants were white, so comparisons by race could not be made and results are not generalizable to non-whites.

Different obesity measures have been associated with clinical and pathological outcomes of RCC. In a recent metaanalysis, higher BMI was inversely associated with mortality (HR 0.57; 95\% Cl 0.43-0.76 when comparing the highest and the lowest BMI). ${ }^{3,16}$ Visceral adiposity has been inconsistently associated with clinicopathological characteristics of RCC. Naya and colleagues reviewed 117 male patients treated with nephrectomy for ccRCC. They found that higher VFA was significantly associated with lower stage disease, fewer events of microvascular invasion, and a higher cumulative cancer-specific survival. ${ }^{13}$ Similarly, Steffens and colleagues noted that a higher than median VFA was associated with lower tumour stage, whereas patients with low VFA suffered more frequently from advanced disease at diagnosis. ${ }^{16}$ More recently, Zhu and colleagues evaluated the association between visceral obesity and Furhman grade in patients with clinical T1a RCC. Unlike previous reports, they demonstrated that high VFA\% was associated with a higher grade of RCC. ${ }^{17}$ Two previous studies evaluated the predic- tive value of visceral adiposity in metastatic RCC patients treated with anti-angiogenic therapy, reporting contradicting results. While one study showed that higher than median VFA values were significantly associated with a shorter progression-free survival and OS, the other demonstrated an opposite correlation. ${ }^{14,15}$ Finally, greater perirenal fat distance, a proxy of visceral fat, was a significant predictor of ccRCC histopathology in clinical T1a tumours. ${ }^{29}$

In the current study, while different obesity measures were significantly associated with hypertension and diabetes, we did not find statistically significant associations between SFA, VFA or VFA\% and grade, stage or OS. However, while not statistically significant, there was a trend toward an association between SFA and OS similar to that seen with BMI. The strong correlation between SFA and BMI, which has been associated with a better prognosis in the current as well as previous studies, suggested that both are similar metrics that may favourably influence survival in RCC patients. Therefore the use of BMI, a parameter which is easier to obtain, may be sufficient when assessing treatment outcome in this group of patients. The difference between our findings and those of previous reports may be partially related to the different study population, which had substantially higher obesity values than previously studied cohorts.

The study limitations include its retrospective nature with the inherent possibility of selection bias. It is also possible that our ability to detect small affect sizes was limited due to the sample size in this study. Due to the small number of deaths in our cohort, we were unable to perform a multivariable analysis to evaluate whether BMI and SFA are independent predictors of clinicopathological outcomes in RCC. In addition, we could not evaluate the significance of visceral adiposity in predicting outcome of patients with metastatic disease and non clear cell RCC histologies, as these were excluded from our study. While our study may suggest previous findings regarding the associations between visceral adiposity and RCC may not be generalizable to Western populations, future studies with larger sample sizes and multiple histological subtypes are needed to clarify the role of body size in predicting tumour histology, characteristics and prognosis in this population. 
Mano et al.

\section{Conclusion}

Although the current study showed that BMI was associated with OS, neither SFA nor VFA were significantly associated with tumour stage, grade or OS in a non-metastatic cCRCC cohort from the United States. While the use of BMI, a more easily obtained measure, may be sufficient when evaluating patient outcomes, further studies in larger cohorts are required to clarify the role of SFA and VFA in Western populations.

Acknowledgement: Supported by the Sidney Kimmel Center for Prostate and Urologic Cancers. Grant support from the Stephen P. Hanson Family Fund Fellowship in Kidney Cancer, The National Cancer Institute T32 CA082088 (AAH) and the Swiss Foundation for Grants in Biology and Medicine (CAK).

Competing interests: Dr. Mano, Dr. Hakimi, Ms. Zabor, Dr. Bury, Dr. Donati, Dr. Karlo, Dr. Bazzi and Dr. Furberg all declare no competing financial or personal interests. Dr. Russo is a consultant for Wilex AG.

This paper has been peer-reviewed.

\section{References}

1. Renehan AG, Tyson M, Egger $M$, et al. Body-mass index and incidence of cancer: A systematic review and meta-analysis of prospective observational studies. Lancet 2008;371:569-78. http://dx.doi. org/10.1016/S0140-6736(08)60269-X

2. Chow WH, Gridley G, Fraumeni JF Jr, et al. Obesity, hypertension, and the risk of kidney cancer in men. N Engl J Med 2000;343:1305-11. http://dx.doi.org/10.1056/NEJM200011023431804

3. Choi $Y$, Park $B$, Jeong $B C$, et al. Body mass index and survival in patients with renal cell carcinoma: $A$ clinical-based cohort and meta-analysis. Int J Cancer 2013;132:625-34. http://dx.doi.org/10.1002/ iic. 27639

4. Hakimi AA, Furberg H, Zabor EC, et al. An epidemiologic and genomic investigation into the obesity paradox in renal cell carcinoma. J Natl Cancer Inst 2013;105:1862-70. http://dx.doi.org/10.1093/inci/dit310

5. Florez $\mathrm{H}$, Castillo-Florez S. Beyond the obesity paradox in diabetes: Fitness, fatness, and mortality. JAMA 2012;308:619-20. http://dx.doi.org/10.1001/jama.2012.9776

6. Doyle SL, Donohoe CL, Lysaght J, et al. Visceral obesity, metabolic syndrome, insulin resistance and cancer. Proc Nutr Soc 2012;71:181-9. http://dx.doi.org/10.1017/S002966511100320X

7. Balentine $\mathrm{CJ}$, Enriquez J, Fisher W, et al. Intra-abdominal fat predicts survival in pancreatic cancer. J Gastrointest Surg 2010;14:1832-7. http://dx.doi.org/10.1007/s11605-010-1297-5

8. Ballian N, Lubner MG, Munoz A, et al. Visceral obesity is associated with outcomes of total mesorectal excision for rectal adenocarcinoma. J Surg Oncol 2012;105:365-70. http://dx.doi.org/10.1002/iso.22031

9. Beddy P, Howard J, McMahon C, et al. Association of visceral adiposity with oesophageal and junctional adenocarcinomas. Br J Surg 2010;97:1028-34. http://dx.doi.org/10.1002/bjs.7100

10. Rickles AS, lannuzzi JC, Mironov 0, et al. Visceral obesity and colorectal cancer: Are we missing the boat with BMI? J Gastrointest Surg 2013;17:133-43; discussion 143.
11. Schapira DV, Clark RA, Wolff PA, et al. Visceral obesity and breast cancer risk. Cancer 1994;74:632-9. http://dx.doi.org/10.1002/1097-0142(19940715)74:2<632::AID-CNCR2820740215>3.0.C0;2-I

12. Sudarshan S, Karam JA, Brugarolas J, et al. Metabolism of kidney cancer: From the lab to clinical practice. Eur Urol 2013;63:244-51. http://dx.doi.org/10.1016/i.eururo.2012.09.054

13. Naya $Y$, Zenbutsu $S$, Araki K, et al. Influence of visceral obesity on oncologic outcome in patients with renal cell carcinoma. Urol Int 2010;85:30-6. http://dx.doi.org/10.1159/000318988

14. Ladoire $S$, Bonnetain $F$, Gauthier $M$, et al. Visceral fat area as a new independent predictive factor of survival in patients with metastatic renal cell carcinoma treated with antiangiogenic agents. Oncologist 2011;16:71-81. http://dx.doi.org/10.1634/theoncologist.2010-0227

15. Steffens $\mathrm{S}$, Grunwald V, Ringe KI, et al. Does obesity influence the prognosis of metastatic renal cell carcinoma in patients treated with vascular endothelial growth factor-targeted therapy? Oncologist 2011;16:1565-71. http://dx.doi.org/10.1634/theoncologist.2011-0213

16. Steffens S, Ringe KI, Schroeer K, et al. Does overweight influence the prognosis of renal cell carcinoma? Results of a multicenter study. Int I Urol 2013;20:585-92. http://dx.doi.org/10.1111/iju.12000

17. Zhu $Y$, Wang HK, Zhang HL, et al. Visceral obesity and risk of high grade disease in clinical tla renal cell carcinoma. J Urol 2013;189:447-53. http://dx.doi.org/10.1016/i.juro.2012.09.030

18. Deurenberg $P$, Yap $M$, van Staveren WA. Body mass index and percent body fat: A meta analysis among different ethnic groups. Int J Obes Relat Metab Disord 1998;22:1164-71. http://dx.doi.org/10.1038/ si.i.jo.0800741

19. Hamdy 0, Porramatikul S, Al-Ozairi E. Metabolic obesity: The paradox between visceral and subcutaneous fat. Curr Diabetes Rev 2006;2:367-73. http://dx.doi.org/10.2174/1573399810602040367

20. American Joint Committee on Cancer: AJCC Cancer Staging Manual. New York, Springer; 2002.

21. Fuhrman $S A$, Lasky LC, Limas C. Prognostic significance of morphologic parameters in renal cell carcinoma. Am J Surg Pathol 1982;6:655-63. http://dx.doi.org/10.1097/00000478-198210000-00007

22. Obesity: Preventing and managing the global epidemic. Report of a WHO consultation. World Health Organ Tech Rep Ser 2000;894:-xii, 1-253.

23. Yoshizumi T, Nakamura T, Yamane M, et al. Abdominal fat: Standardized technique for measurement at CT. Radiology 1999;211:283-6. http://dx.doi.org/10.1148/radiology.211.1.r99ap15283

24. Barboriak DP, Padua AO, York GE, et al. Creation of DICOM-aware applications using ImageJ. J Digit Imaging 2005;18:91-9. http://dx.doi.org/10.1007/s10278-004-1879-4

25. Ibrahim MM. Subcutaneous and visceral adipose tissue: Structural and functional differences. Obes Rev 2010;11:11-8. htrp://dx.doi.org/10.1111/i.1467-789X.2009.00623.x

26. Shuster $A$, Patlas $M$, Pinthus JH, et al. The clinical importance of visceral adiposity: A critical review of methods for visceral adipose tissue analysis. Br J Radiol 2012;85:1-10. http://dx.doi.org/10.1259/ $\mathrm{bir} / 38447238$

27. Britton KA, Massaro JM, Murabito JM, et al. Body fat distribution, incident cardiovascular disease, cancer, and all-cause mortality. J Am Coll Cardiol 2013;62:921-5. http://dx.doi.org/10.1016/i. jacc.2013.06.027

28. Camhi SM, Bray GA, Bouchard C, et al. The relationship of waist circumference and BMI to visceral, subcutaneous, and total body fat: sex and race differences. Obesity (Silver Spring) 2011;19:402-8. http://dx.doi.org/10.1038/oby.2010.248

29. Okhunov Z, Mues AC, Kline M, et al. Evaluation of perirenal fat as a predictor of cTla renal cortical neoplasm histopathology and surgical outcomes. J Endourol 2012;26:911-6. http://dx.doi.org/10.1089/ end.2011.0599

Correspondence: Dr. Paul Russo, Urology Service, Department of Surgery, Memorial Sloan Kettering Cancer Center, 1275 York Ave.; New York, NY 10065; russop@mskcc.org; and Dr. Helena Furberg, Department of Epidemiology and Biostatistics, Memorial Sloan Kettering Cancer Center, 1275 York Ave., New York, NY 10065; furberga@mskcc.org 